\title{
The Bayley-III Adaptive Behavior and Social-Emotional Scales as Important Predictors of Later School-Age Out- comes of Children Born Preterm
}

\author{
Jungha Yun*, Ee-Kyung Kim*,†, Seung Han Shin", ${ }^{*}$, Han-Suk Kim, ${ }^{* \dagger}$, Jin A Lee ${ }^{\dagger}$, Eun Sun Kim, and Hye Jeong Jin* \\ Department of Pediatrics*, Seoul National University Children's Hospital, Seoul National University College of Medicine, Seoul, Korea \\ Department of Pediatrics ${ }^{\dagger}$, Seoul National University College of Medicine, Seoul, Korea \\ Department of Pediatrics ${ }^{\ddagger}$, Kangwon National University School of Medicine, Chuncheon, Korea
}

\section{ABSTRACT}

Purpose: We aim to assess the Bayley Scales of Infant and Toddler Development, third edition (Bayley-III), Adaptive Behavior (AB) and Social-Emotional (SE) scales at 18 to 24 months of corrected age (CA) to examine their associations with school-age cognitive and behavioral outcomes in children born preterm.

Methods: Eighty-eight infants born with a very low birth weight $(<1,500 \mathrm{~g})$ or a gestational age of less than 32 weeks who were admitted to the neonatal intensive care unit from 2008 to 2009 were included. Of the 88 children who completed school-age tests at 6 to 8 years of age, 37 were assessed using the Bayley-III, including the $\mathrm{AB}$ and SE scales, at 18 to 24 months of CA. Correlation, cross-tabulation, and receiver operating characteristic analyses were performed to assess the longitudinal associations. Results: A significant association was observed between communication scores on the Bayley-III AB scale at 18 to 24 months of CA and the Korean version of the Wechsler Intelligence Scale for Children (K-WISC) full-scale intelligence quotient (FSIQ) at school age ( $r=0.531)$. The total behavior problem scores of the Korean version of the Child Behavior Checklist (K-CBCL) at school age were significantly negatively related to the Bayley-III SE and AB scales but not to the cognitive, language, or motor scales.

Conclusion: Our findings encourage $\mathrm{AB}$ and $\mathrm{SE}$ assessments during the toddler stage and have important implications for the early identification of children in need of intervention and the establishment of guidelines for follow-up with high-risk infants.

Key Words: Bayley-III, Social-emotional, Adaptive behavior, Preterm, School-age follow-up

\section{INTRODUCTION}

Infants born preterm are at greater risk of neurodevelopmental impairment than infants
Received: 24 July 2018

Revised: 16 October 2018

Accepted: 21 October 2018

Correspondence to: Ee-Kyung Kim

Department of Pediatrics, Seoul National University College of Medicine, 101 Daehak-ro, Jongno-gu, Seoul 03080, Korea

Tel: +82-2-2072-3628

Fax: +82-2-743-3455

E-mail: kimek@snu.ac.kr

Copyright(c)

By Korean Society of Neonatology.

All right reserved.

This is an Open-Access article distributed under the terms of the Creative Commons Attribution Non-Commercial License (http://creativecommons.org/licenses/ by-nc/4.0), which permits unrestricted non-commercial use, distribution, and reproduction in any medium, provided the original work is properly cited. 
born at term ${ }^{1,2)}$. The Bayley Scales of Infant and Toddler Development, third edition (Bayley-III, 2006), are frequently used to assess infant neurodevelopment. The Bayley-III includes separate composite scores for cognitive, language, and motor scales. Additionally, primary caregiver-reported questionnaires are incorporated to assess Social-Emotional (SE) and Adaptive Behavior (AB) aspects. Arpi and Ferrari ${ }^{3)}$ found that SE difficulties in children born preterm predicted behavior problems and that the prevalence of one or more behavior problems was higher in children born preterm than in children born at term. Furthermore, they found that behavior problems tended to co-occur with disabilities in the cognitive, motor, and language domains.

Both the Bayley-II and III are used to assess neurodevelopmental outcome of children in Korea. To date, few studies have reported results related to the Bayley-III SE and AB scales ${ }^{4-7)}$, with most studies focusing on the cognitive, language, and motor scales of the Bayley-III or the mental developmental index (MDI) and the psychomotor developmental index (PDI) of the previous edition of the Bayley Scales of Infant Development, second edition (BSID-II) ${ }^{8,9}$. The Bayley-III SE and AB scales have the potential to provide more clinically useful information for the prediction of neurodevelopmental outcomes related to cognitive function and behavior problems at school age and to contribute to the development of early intervention programs to improve impairment.

In the current study, we aimed to assess the Bayley-III AB and SE scales at 18 to 24 months of corrected age (CA) to examine the association with school-age cognitive and behavioral outcomes of preterm school-aged children. We focused on the Bayley-III $\mathrm{AB}$ scale, particularly, the conceptual adaptive domain, which summarizes performance across the communication, functional pre-academics, and self-direction skill areas, as higher cognitive control is presumed to have a more important impact than earlier stages of development.

\section{MATERIALS AND METHODS}

\section{Study design}

A total of 88 infants with very low birth weights or gestational ages less than 32 weeks at birth who were admitted to the neonatal intensive care unit (NICU) from January 2008 to December 2009 participated in the neurodevelopmental follow-up study of preterm infants at school age. Infants with major congenital anomalies, chromosomal abnormalities, and incomplete medi cal records were excluded. Completion of the Bayley-III was recommended for all infants enrolled in the follow-up program, and permission was obtained from the parents of 37 participants (42\%) to reassess the children using the Bayley-III at 18 to 24 months of CA. Participants were also assessed at 6 to 8 years of age when they completed school-age tests, including the Korean version of the Wechsler Intelligence Scale for Children (K- WISC) and the Korean version of the Child Behavior Checklist (K-CBCL). This study was approved by the Institutional Research Ethics Committee of Seoul National University Hospital, Seoul, Republic of Korea (IRB No. 1507-094-689). The consent form was waived.

\section{Data collection and definitions}

The following clinical data of the infants were collected: birth weight, gestational age, whether infants were small for gestational age (birth weight $<10$ th percentile for age), and prenatal steroid administration (any dose of corticosteroids administered during pregnancy). The comorbidities of preterm infants were assessed, including blood culture-proven sepsis (determined by at least one positive blood culture and clinical signs of infection), necrotizing enterocolitis (according to the modified Bell's criteria) ${ }^{10)}$, periventricular leukomalacia, retinopathy of prematurity, and bronchopulmonary dysplasia (BPD), which was defined based on the National Institutes of Health consensus definition and graded as mild, moderate, or severe according to the fraction of inspired oxygen $\left(\mathrm{FiO}_{2}\right)$ or positive pressure ventilation $(\mathrm{PPV})^{11)}$. Mild BPD was defined as breathing room air, moderate BPD was defined as an $\mathrm{FiO}_{2}<0.30$, and severe BPD was defined as an $\mathrm{FiO}_{2}$ $\geq 0.30$ or PPV at 36 weeks of postmenstrual age. Cerebral palsy (abnormal tone and delays in motor control and function), a K-WISC-IV full-scale intelligence quotient (FSIQ) $<70$, blindness (visual acuity $<20 / 200$ in the better eye), and deafness (hearing loss requiring amplification or a more severe condition) were assessed at school age. Social environmental data were collected at the time of testing and included maternal age at delivery, maternal education, and household income.

\section{Neurodevelopmental assessment}

\section{1) Bayley-III}

Preterm infants discharged to their homes were evaluated at 18 to 24 months of CA by one neonatologist in the neonatology outpatient clinic. Assessments included the composite scores 
on the Bayley-III ${ }^{12)}$. The Bayley-III assesses infant and toddler development across five domains: cognitive, language, motor, $\mathrm{SE}$, and $\mathrm{AB}$. Assessments of the cognitive, language, and motor domains are conducted using items administered to the child; assessments of the SE and $\mathrm{AB}$ domains are conducted using the primary caregiver's responses to a questionnaire. The questionnaire comprises the SE scale (35 items) and the AB scale. The areas measured within the $\mathrm{AB}$ scale include conceptual (communication, functional pre-academics, self-direction), social (social, leisure), and practical (community use, home living, health and safety, self-care) domains. The conceptual adaptive domain is calculated by adding the communication (25 items), functional pre-academics (22 items), and self-direction (25 items) skill area scores. Children whose composite scores were less than 85 (1 standard deviation [SD] below average) on the cognitive, language, motor, $\mathrm{SE}$, and $\mathrm{AB}$ scales and those whose scaled scores were less than 7 (1 SD below average) on the BayleyIII communication, functional pre-academics, and self-direction skill areas were considered impaired (Bayley-III low group).

\section{2) School-age tests}

(1) Cognitive test: K-WISC-IV

General intellectual ability was evaluated using the Korean version of the K-WISC-IV ${ }^{13,14)}$. The FSIQ was used to assess general intelligence, and the index scores of the four subdomains were evaluated to obtain more specific cognitive information. The verbal comprehension index (VCI) was used as a measure of the knowledge of word meanings and verbal reasoning abilities, the perceptual reasoning index (PRI) was used as a measure of visuoconstructional skills and visual reasoning abilities, the working memory index (WMI) was used as a measure of immediate and working memory, and the processing speed index (PSI) was used as a measure of speed and accuracy of information processing. The FSIQ was standardized by age, with a mean of 100 and an SD of 15.

(2) Behavioral test: K-CBCL

Behavioral outcomes were evaluated with the K-CBCL, which is a frequently used questionnaire with 119 items that provides parent-reported data on behavioral problems in children ${ }^{15-17)}$. The K-CBCL has a total of eight subscales (withdrawn, somatic complaints, anxious/depressed, and social, thought, attention, delinquency, and aggression problems) that are classified as internalizing behavior problems (withdrawn, somatic complaints, and anxious/depressed) and externalizing behavior problems (attention, delinquency, and aggression problems). Total behavior problem scores were calculated by summing the scores obtained for each item. The raw score of the K-CBCL can be converted to a T-score with a mean of 50 points and an SD of 10 points. High scores suggest more problems, and T-scores of 60 or more are considered in the clinical range and are indicative of behavior problems ${ }^{18)}$.

\section{Statistical analysis}

The data analysis was performed using SPSS version 20.0 for Windows (IBM Co., Armonk, NY, USA). A $t$-test was used to compare Bayley-III scores with K-WISC-IV scores. Proportions were evaluated using the chi-square test and Fisher's exact test. Pearson's correlation was used to analyze the correlations among the five scales of the Bayley-III, K-WISC, and K-CBCL. We examined the sensitivity, specificity, positive predictive value, and negative predictive value by analyzing receiver operating characteristic (ROC) curves. $P$-values $<0.05$ were considered statistically significant. Data are presented as the mean $\pm S D$, medians and ranges, or rates.

\section{RESULTS}

\section{Demographic characteristics}

Of the 37 children who completed both the Bayley-III at 18 to 24 months of CA and the school-age tests, including the K-WISCIV and K-CBCL, at 6 to 8 years of age, two were not eligible for follow-up, and six did not complete the full assessment. The remaining 29 children constituted the study population (Figure 1). Table 1 shows the characteristics of the sample during the NICU stay and at the school-age follow-up. The mean gestational age was 27.5 weeks, and the mean birth weight was $927 \mathrm{~g}$. Two children were diagnosed with cerebral palsy (5.7\%), and none were blind or deaf. The mean K-WISC-IV FSIQ score was 82.4 $(\mathrm{SD}=26.8)$ and $26.7 \%(\mathrm{n}=8)$ had K-WISC-IV FSIQ scores lower than 70 (2SD).

\section{Correlations and comparisons of the Bayley-III subscales}

The correlations among the Bayley-III cognitive, language, motor, SE, and $\mathrm{AB}$ scales were between 0.360 and 0.781 . The SE scale did not exhibit a significant correlation with the Cognitive scale but had mild to moderate correlations with the language ( $r=0.474, P<0.01)$ and motor scales $(r=0.360, P<0.05)$. The AB 


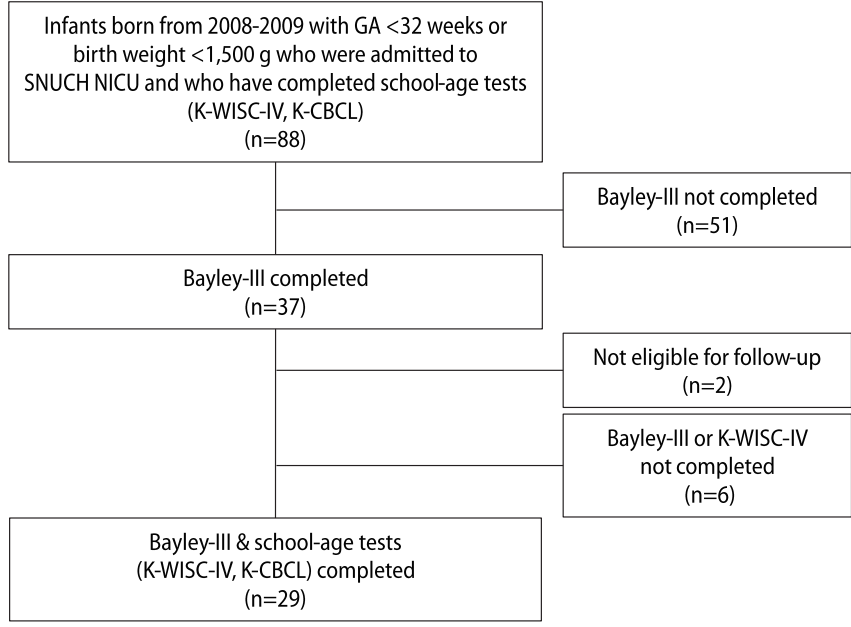

Figure 1. Flow chart of the study. Abbreviations: GA, gestational age; SNUCH, Seoul National University Children's Hospital; NICU, neonatal intensive care unit; K-WISC, Korean version of the Wechsler Intelligence Scale for Children; K-CBCL, Korean version of the Child Behavior Checklist; Bayley-III, Bayley Scales of Infant and Toddler Development, third edition.

Table 1. Demographic and Clinical Characteristics of the Children

\begin{tabular}{|c|c|}
\hline Characteristic & Value \\
\hline \multicolumn{2}{|c|}{ Patient characteristics during NICU stay (n=37) } \\
\hline Gestational age (wk) & $27.5 \pm 2.3$ \\
\hline Born <28 wk & $22(59.5)$ \\
\hline Birth weight (g) & $927 \pm 282$ \\
\hline Weight $<1,000 \mathrm{~g}$ & $28(75.7)$ \\
\hline \multicolumn{2}{|c|}{ Patient outcome in infancy $(\mathrm{n}=37)$} \\
\hline Age at the Bayley-III (mo) & $19.6 \pm 1.5$ \\
\hline \multicolumn{2}{|l|}{ Bayley-III composite score } \\
\hline Cognitive & $97.6 \pm 12.9$ \\
\hline Language & $90.9 \pm 12.8$ \\
\hline Motor & $96.3 \pm 12.8$ \\
\hline Social-emotional & $95.3 \pm 20.3$ \\
\hline Adaptive behavior & $81.3 \pm 17.0$ \\
\hline Cerebral palsy & $2(5.4)$ \\
\hline Blindness or deafness & 0 \\
\hline \multicolumn{2}{|c|}{ Patient outcome at early school age (n=29) } \\
\hline Age at school-age test (yr) & $7.3 \pm 0.4$ \\
\hline K-WISC-IV FSIQ & $82.4 \pm 26.8$ \\
\hline \multicolumn{2}{|l|}{ K-CBCL T-score } \\
\hline Internalizing problems & $55.6 \pm 8.8$ \\
\hline Externalizing problems & $52.7 \pm 9.1$ \\
\hline
\end{tabular}

Values are expressed as mean \pm standard deviation or number (\%). Abbreviations: NICU, neonatal intensive care unit; Bayley-III, Bayley Scales of Infant and Toddler Development, third edition; K-WISC, Korean version of the Wechsler Intelligence Scale for Children; FSIQ, full-scale intelligence quotient; K-CBCL, Korean version of the Child Behavior Checklist. scale exhibited significant positive correlations with the cognitive, language, and motor scales and showed stronger positive correlations than the correlation between the SE scale and these three subscales of the Bayley-III ( 0.550 vs. 0.474 and 0.559 vs. 0.360 ).

\section{Correlations of the Bayley-III subscales with school-age tests (K-WISC-IV and K-CBCL)}

The correlation coefficients of the Bayley-III scales with the K-WISC-IV FSIQ were between 0.356 and 0.612 (Table 2). Among the three conceptual adaptive domains (communication, functional pre-academics, and self-direction) in the Bayley-III $\mathrm{AB}$ scale, the communication scaled scores had a moderate correlation with the K-WISC-IV FSIQ $(r=0.531, P<0.01)$. This result showed that the correlation between the communication skill area and the K-WISC-IV FSIQ was comparable to that of the cognitive scale of the Bayley-III with the K-WISC-IV FSIQ (0.531 vs. 0.612, each $P<0.01$ ). The total behavior problem scores of the K-CBCL were significantly negatively associated with the BayleyIII SE and AB scales but not with the cognitive, language, or motor scales $(P<0.05)$. The internalizing behavior problem scores of the K-CBCL exhibited a significant moderate correlation with the Bayley-III SE scale $(r=-0.368, P<0.05)$. The withdrawn score, one of the internalizing behavior problems, had a significantly stronger correlation with the Bayley-III SE scale than any other Bayley-III scale $(r=-0.416, P<0.01)$.

Table 2. Correlation of the Bayley-III Subscales with the SchoolAge Tests (K-WISC-IV and K-CBCL) $(\mathrm{n}=29)$

\begin{tabular}{|c|c|c|c|c|}
\hline Bayley-III & FSIQ & $\begin{array}{l}\text { Internalizing } \\
\text { problems }\end{array}$ & $\begin{array}{c}\text { Externalizing } \\
\text { problems }\end{array}$ & $\begin{array}{c}\text { Total behavior } \\
\text { problems }\end{array}$ \\
\hline Cognitive & $0.612^{*}$ & -0.309 & -0.204 & -0.292 \\
\hline Language & $0.477^{*}$ & -0.201 & -0.243 & -0.237 \\
\hline Motor & $0.523^{*}$ & -0.302 & -0.19 & -0.25 \\
\hline Social-emotional & $0.361^{\dagger}$ & $-0.368^{\dagger}$ & -0.266 & $-0.365^{\dagger}$ \\
\hline Adaptive behavior & $0.48^{*}$ & -0.342 & -0.33 & $-0.467^{*}$ \\
\hline Communication & $0.531^{*}$ & -0.34 & -0.188 & -0.349 \\
\hline $\begin{array}{l}\text { Functional pre- } \\
\text { academics }\end{array}$ & $0.359^{\dagger}$ & -0.126 & -0.177 & -0.149 \\
\hline Self-direction & $0.356^{\dagger}$ & -0.303 & -0.26 & $-0.421^{\dagger}$ \\
\hline
\end{tabular}

${ }^{*} P<0.01 ;{ }^{\dagger} P<0.05$.

Abbreviations: Bayley-III, Bayley Scales of Infant and Toddler Development, third edition; K-WISC, Korean version of the Wechsler Intelligence Scale for Children; K-CBCL, Korean version of the Child Behavior Checklist; FSIQ, full-scale intelligence quotient. 


\section{Predictive ability of the Bayley-III subscales for the school- age tests (K-WISC-IV and K-CBCL)}

The mean differences in the Bayley-III scores between scores 1 SD below and above or equal to the K-WISC-IV FSIQ score of 85 are shown in Table 3. The sensitivity of low scores (<-1 SD) on the Bayley-III subscales at 18 to 24 months of CA for predicting mild impairment on the K-WISC-IV FSIQ (<-1 SD) at school age was low, but the specificity was high (Table 4 ). The Bayley-III language and $\mathrm{AB}$ scales showed significantly high specificity and areas under curves (AUCs), whereas the Cognitive scale had a lower AUC and did not exhibit a significant predictive ability. The AUC of the Bayley-III AB scale was a fair method for predicting

Table 3. Comparisons of Mean Composite Scores on the BayleyIII Subscales at 18 to 24 Months of CA for the Mild Impairment Group on the K-WISC-IV FSIQ at School Age (N=29, Low Group $<-1 \mathrm{SD})$

\begin{tabular}{lcccc}
\hline Bayley-III & $\begin{array}{c}\text { FSIQ }<85 \\
(\mathrm{n}=13)\end{array}$ & $\begin{array}{c}\text { FSIQ } \geq 85 \\
(\mathrm{n}=16)\end{array}$ & $\begin{array}{c}\text { Composite } \\
\text { score } \\
\text { difference }\end{array}$ & $P$-value \\
\hline Cognitive & $90.9 \pm 10.5$ & $105.8 \pm 10.8$ & 14.8 & 0.001 \\
Language & $86.6 \pm 13.3$ & $96.2 \pm 10.1$ & 9.7 & 0.040 \\
Motor & $91.1 \pm 12.2$ & $102.7 \pm 10.8$ & 11.6 & 0.012 \\
Social-emotional & $90.3 \pm 22.5$ & $101.5 \pm 16.0$ & 11.2 & 0.142 \\
Adaptive behavior & $74.3 \pm 16.1$ & $90.0 \pm 14.2$ & 15.8 & 0.010 \\
\hline
\end{tabular}

Values are expressed as mean \pm standard deviation unless otherwise stated.

Abbreviations: Bayley-III, Bayley Scales of Infant and Toddler Development, third edition; CA, corrected age; K-WISC, Korean version of the Wechsler Intelligence Scale for Children; FSIQ, full-scale intelligence quotient; SD, standard deviation. mild impairment on the K-WISC-IV FSIQ (AUC, $0.767 ; P<0.01$ ). In the communication skill area, one of the conceptual adaptive domains of the Bayley-III AB scale was acceptable for predicting the K-WISC-IV FSIQ results (AUC, $0.680 ; P<0.050$ ). The sensitivity and specificity of low scaled scores (<-1 SD) on the Bayley-III communication area at 18 to 24 months of $\mathrm{CA}$ for predicting mild problems on the K-CBCL (>1 SD) at school age were reasonable, and the negative predictive value was high (Table 5). The BayleyIII communication scaled score at 18 to 24 months of CA had a significant AUC for predicting the subscales of the K-CBCL at school age (AUC, 0.700 to $0.879 ; P<0.05$ ) using a communication scaled score cutoff of $<-1 \mathrm{SD}(<7)$. This method was a good predictor of the K-CBCL aggression problems score, which had the highest AUC among the eight subscale scores (AUC, 0.879; $P<0.01)$.

\section{DISCUSSION}

In this study, we assessed the Bayley-III SE and AB scales, which were added to the revised Bayley assessment in its third edition ${ }^{12)}$. The Bayley-III SE and AB scales showed mild to moderate correlations with the cognitive, language, and motor scales. For prediction of later neurodevelopmental outcomes, we found that the Bayley-III SE and AB scales at 18 to 24 months of CA were significantly associated with school-age cognitive and behavioral outcomes on the K-WISC-IV and K-CBCL. Treyvaud et al. ${ }^{19)}$ demonstrated that internalizing problems at 2 years of

Table 4. Analytical Values of Low Scores on the Bayley-III Subscales at 18 to 24 Months of CA for Predicting Mild Impairment on the K-WISC-IV FSIQ at School Age ( $\mathrm{n}=29$, Low Group $<-1$ SD)

\begin{tabular}{|c|c|c|c|c|c|}
\hline \multirow{2}{*}{ Bayley-III low group (<-1 SD) } & \multicolumn{5}{|c|}{ 95\% Confidence interval } \\
\hline & AUC & Sensitivity & Specificity & PPV & NPV \\
\hline Cognitive & $0.524(0.331-0.711)$ & $12.5(1.6-38.3)$ & $92.3(64.0-99.8)$ & $66.7(16.9-95.2)$ & $46.2(40.2-52.2)$ \\
\hline Language & $0.680 *(0.482-0.840)$ & $43.8(19.8-70.1)$ & $92.3(64.0-99.8)$ & $87.5(49.6-98.0)$ & $57.1(45.7-67.9)$ \\
\hline Motor & $0.625^{*}(0.427-0.797)$ & $25.0(7.3-52.4)$ & 100.0 & 100.0 & $52.0(44.9-59.0)$ \\
\hline Social-emotional & $0.649 *(0.451-0.816)$ & $37.5(15.2-64.6)$ & $92.3(64.0-99.8)$ & $85.7(45.1-97.8)$ & $54.5(44.3-64.4)$ \\
\hline Adaptive behavior & $0.767^{\dagger}(0.574-0.903)$ & $68.8(41.3-89.0)$ & $84.6(54.6-98.1)$ & $84.6(59.6-95.4)$ & $68.8(50.6-82.5)$ \\
\hline Communication & $0.680 *(0.482-0.840)$ & $43.8(19.8-70.1)$ & $92.3(64.0-99.8)$ & $87.5(49.6-98.0)$ & $57.1(45.7-67.9)$ \\
\hline Functional pre-academics & $0.642(0.443-0.810)$ & $43.8(19.8-70.1)$ & $84.6(54.6-98.1)$ & $77.8(46.6-93.4)$ & $55.0(42.8-66.6)$ \\
\hline Self-direction & $0.673^{*}(0.475-0.835)$ & $50.0(24.7-75.3)$ & $84.6(54.6-98.1)$ & $80.0(50.5-94.0)$ & $57.9(44.4-70.3)$ \\
\hline
\end{tabular}

${ }^{*} P<0.05 ;{ }^{\dagger} P<0.01$.

Abbreviations: Bayley-III, Bayley Scales of Infant and Toddler Development, third edition; CA, corrected age; K-WISC, Korean version of the Wechsler Intelligence Scale for Children; FSIQ, full-scale intelligence quotient; SD, standard deviation; AUC, area under curve; PPV, positive predictive value; NPV, negative predictive value. 
Table 5. Analytical Values of Low Bayley-III Communication Scaled Scores at 18 to 24 Months of CA for Predicting Mild Problems on the K-CBCL at School Age (n=29, Bayley-III Low Group <-1 SD, Problems on the K-CBCL >1 SD)

\begin{tabular}{lccccc}
\hline \multirow{2}{*}{$\begin{array}{l}\text { Communication low group } \\
(<-1 \text { SD })\end{array}$} & \multicolumn{4}{c}{$95 \%$ Confidence interval } \\
\cline { 2 - 6 } & AUC & Sensitivity & Specificity & PPV & NPV \\
\hline Somatic complaints & $0.860^{*}(0.718-0.897)$ & $100.0(15.8-100.0)$ & $88.9(70.8-97.6)$ & $40.0(18.7-66.0)$ & 100.0 \\
Anxious/depressed & $0.708^{\dagger}(0.512-0.861)$ & $60.0(14.7-94.7)$ & $70.8(48.9-87.4)$ & $30.0(14.2-52.5)$ & $89.5(73.8-96.2)$ \\
Social problems & $0.766^{\dagger}(0.573-0.902)$ & $71.4(29.0-96.3)$ & $86.4(65.1-97.1)$ & $62.5(34.5-84.1)$ & $90.5(74.4-96.9)$ \\
Attention problems & $0.700^{\dagger}(0.502-0.855)$ & $75.0(19.4-99.4)$ & $80.0(59.3-93.2)$ & $37.5(18.6-61.2)$ & $95.2(78.4-99.1)$ \\
Aggressive problems & $0.879^{*}(0.761-0.989)$ & $100.0(29.2-100.0)$ & $80.8(60.6-93.4$ & $37.5(21.4-56.9)$ & 100.0 \\
\hline
\end{tabular}

${ }^{*} P<0.01 ;{ }^{\dagger} P<0.05$.

Abbreviations: Bayley-III, Bayley Scales of Infant and Toddler Development, third edition; CA, corrected age; K-CBCL, Korean version of the Child Behavior Checklist; SD, standard deviation; AUC, area under curve; PPV, positive predictive value; NPV, negative predictive value.

age were strongly associated with the emotional symptoms of 5 -year-old children born very preterm. The tendency toward more withdrawn behavior at school age may be linked to lower rates of social interactions ${ }^{20}$. The results of this study showed that higher rates of internalizing problems, such as anxiety, depression, and social isolation, in school-age children born preterm were predicted by lower SE composite scores at 18 to 24 months of CA. Therefore, monitoring SE development for early intervention may prevent emotional problems related to social interactions from becoming severe at school age.

Furthermore, we found that among the three conceptual adaptive domains in the Bayley-III AB scale, the communication skill area was a strong predictor of cognitive function and behavior problems. This area had a concerningly low sensitivity but high specificity and a reasonable AUC for predicting neurodevelopmental impairment using a communication scaled score $<-1$ SD as a cutoff. Two studies have identified that communication deficits are likely to increase externalizing behavior problems because communication-delayed children demonstrate less social maturity and intellectual development than their peers, which can affect their relationships with their peers and their school performance ${ }^{21,22)}$. Brassart and Schelstraete ${ }^{23)}$ proposed that early interventions could decrease child behavior problems by enhancing the parents' verbal responsiveness and children's communication abilities. Speech and language delays are common in preterm children compared with children born at full-term, with comprehension problems showing earlier and production problems appearing later ${ }^{24,25}$. This has important implications for developing communication functions during the toddler stage, preventing behavior problems, and improving cognitive outcomes. This finding also suggests that the early identification of children in need of intervention can improve out- comes.

The main limitation of the current study is that our observations are restricted to a small sample. Therefore, these findings cannot be generalized to broader populations and clinical applications based on this study alone. Future research is needed to assess the clinical implications of the Bayley-III SE and AB scales in sufficiently large samples. Two children with cerebral palsy were included in the study. They achieved low scores on the Bayley-III and school-age tests, but their scores did not impact the main outcomes of our study. The neurodevelopmental impairments evaluated by the Bayley-III SE and AB scales, which are derived from primary caregiver-reported questionnaires, possibly underestimate the problem in some studies due to the tendency of caregivers to exaggerate their children's performances in their reports ${ }^{6,26)}$. However, through questionnaires, these two scales increase the role of the primary caregiver in the assessment process. Given the potential for the clinical utility of these two scales, particularly the Communication skill area for predicting later neurodevelopmental outcomes, future research is needed to identify more appropriate clinical properties of the areas assessed and whether they underestimate or overestimate neurodevelopmental delays compared with the estimates provided by other scales. Finally, this study did not examine the influences of maternal mental health factors. It has been found that maternal mental health factors of depression, anxiety, or stress are associated with impaired social-emotional development in very preterm infants at the age of 2 years ${ }^{7,27)}$. Further longitudinal research examining how maternal mental problems influence emotional well-being in child born preterm may be helpful.

In conclusion, the Bayley-III SE and AB scales at 18 to 24 months of CA showed significant correlations with school-age cognitive and behavioral outcomes of children born preterm, 
suggesting that $\mathrm{SE}$ and $\mathrm{AB}$ questionnaires are a useful tool in the neurodevelopmental follow-up study at school age. Optimal development of communication function during the toddler stage could be a strategy worth adopting to prevent behavior problems and improve cognitive outcomes.

\section{CONFLICT OF INTEREST}

No potential conflict of interest relevant to this article was reported.

\section{ACKNOWLEDGMENTS}

This work was supported under the framework of international cooperation program managed by National Research Foundation of Korea (No. NRF-2017R1D1A1B04030931, 2017) and the Basic Science Research Program through the National Research Foundation of Korea funded by the Ministry of Education (HI14C3451, 2012).

\section{REFERENCES}

1. Saigal S, Doyle LW. An overview of mortality and sequelae of preterm birth from infancy to adulthood. Lancet 2008;371:2619.

2. Bhutta AT, Cleves MA, Casey PH, Cradock MM, Anand KJ. Cognitive and behavioral outcomes of school-aged children who were born preterm: a meta-analysis. JAMA 2002;288:72837.

3. Arpi E, Ferrari F. Preterm birth and behaviour problems in infants and preschool-age children: a review of the recent literature. Dev Med Child Neurol 2013;55:788-96.

4. Fernandes LV, Goulart AL, Santos AM, Barros MC, Guerra CC, Kopelman BI. Neurodevelopmental assessment of very low birth weight preterm infants at corrected age of 18-24 months by Bayley III scales. J Pediatr (Rio J) 2012;88:471-8.

5. Huang JH, Huang HL, Chen HL, Lin LC, Tseng HI, Kao TJ. Inattention and development of toddlers born in preterm and with low birth weight. Kaohsiung J Med Sci 2012;28:390-6.

6. Velikos K, Soubasi V, Michalettou I, Sarafidis K, Nakas C, Papadopoulou V, et al. Bayley-III scales at 12 months of corrected age in preterm infants: patterns of developmental performance and correlations to environmental and biological influences.
Res Dev Disabil 2015;45-46:110-9.

7. Gray PH, Edwards DM, Hughes IP, Pritchard M. Social-emotional development in very preterm infants during early infancy. Early Hum Dev 2018;121:44-8.

8. Spencer-Smith MM, Spittle AJ, Lee KJ, Doyle LW, Anderson PJ. Bayley-III cognitive and language scales in preterm children. Pediatrics 2015;135:e1258-65.

9. Girsen AI, Do SC, El-Sayed YY, Hintz SR, Blumenfeld YJ. Association between small-for-gestational age and neurocognitive impairment at two years of corrected age among infants born at preterm gestational ages: a cohort study. J Perinatol 2017;37:958-62.

10. Bell MJ, Ternberg JL, Feigin RD, Keating JP, Marshall R, Barton L, et al. Neonatal necrotizing enterocolitis. Therapeutic decisions based upon clinical staging. Ann Surg 1978;187:1-7.

11. Jobe AH, Bancalari E. Bronchopulmonary dysplasia. Am J Respir Crit Care Med 2001;163:1723-9.

12. Bayley N. Bayley scales of infant and toddler development. 3rd ed. San Antonio: Harcourt Assessment, 2006.

13. Kwak K, Oh S, Kim C. Manual for Korean Wechsler Intelligence Scale for Children-IV (K-WISC-IV)-manual. Seoul: Hakjisa, 2011.

14. Park S, Cho SC, Hong YC, Kim JW, Shin MS, Yoo HJ, et al. Environmental tobacco smoke exposure and children's intelligence at 8-11 years of age. Environ Health Perspect 2014;122: 1123-8.

15. Achenbach TM. Manual for the child behavior checklist/4-18 and 1991 profile. Burlington: University of Vermont Department of Psychiatry, 1991.

16. Oh KJ, Lee HL, Hong KE, Ha EH. Development of Korean version of Child Behavior Checklist (K-CBCL). Seoul: Korean Research Foundation Report, 1997.

17. Kim JI, Kang YH, Lee JM, Cha J, Park YH, Kweon KJ, et al. Resting-state functional magnetic resonance imaging investigation of the neural correlates of cognitive-behavioral therapy for externalizing behavior problems in adolescent bullies. Prog Neuropsychopharmacol Biol Psychiatry 2018;86:193-202.

18. Nolan TM, Bond L, Adler R, Littlefield L, Birleson P, Marriage $\mathrm{K}$, et al. Child Behaviour Checklist classification of behaviour disorder. J Paediatr Child Health 1996;32:405-11.

19. Treyvaud K, Doyle LW, Lee KJ, Roberts G, Lim J, Inder TE, et al. Social-emotional difficulties in very preterm and term 2 year olds predict specific social-emotional problems at the age of 5 years. J Pediatr Psychol 2012;37:779-85.

20. Spittle AJ, Treyvaud K, Doyle LW, Roberts G, Lee KJ, Inder TE, et al. Early emergence of behavior and social-emotional problems in very preterm infants. J Am Acad Child Adolesc Psychiatry 2009;48:909-18.

21. Monopoli WJ, Kingston S. The relationships among language 
ability, emotion regulation and social competence in secondgrade students. Int J Behav Dev 2012;36:398-405.

22. Ketelaars MP, Cuperus J, Jansonius K, Verhoeven L. Pragmatic language impairment and associated behavioural problems. Int J Lang Commun Disord 2010;45:204-14.

23. Brassart E, Schelstraete MA. Enhancing the communication abilities of preschoolers at risk for behavior problems. Infant Young Child 2015;28:337-54.

24 . Vohr B. Speech and language outcomes of very preterm infants. Semin Fetal Neonatal Med 2014;19:78-83.

25. Sansavini A, Pentimonti J, Justice L, Guarini A, Savini S, Alessan- droni $\mathrm{R}$, et al. Language, motor and cognitive development of extremely preterm children: modeling individual growth trajectories over the first three years of life. J Commun Disord 2014; 49:55-68.

26. Miller SA, Manhal M, and Mee LL. Parental beliefs, parental accuracy, and children's cognitive performance: a search for causal relations. Dev Psychol 1991;27:267-76.

27. Treyvaud K, Anderson VA, Lee KJ, Woodward LJ, Newnham C, Inder TE, et al. Parental mental health and early social-emotional development of children born very preterm. J Pediatr Psychol 2010;35:768-77. 